\title{
SeaConditions: a web and mobile service for safer professional and recreational activities in the Mediterranean Sea
}

Giovanni Coppini ${ }^{1}$, Palmalisa Marra ${ }^{2}$, Rita Lecci $^{1}$, Nadia Pinardi ${ }^{1,3}$, Sergio Creti ${ }^{1}$, Mario Scalas $^{2}$, Luca Tedesco $^{2}$, Alessandro D'Anca ${ }^{1}$, Leopoldo Fazioli ${ }^{4}$, Antonio Olita ${ }^{4}$, Giuseppe Turrisi ${ }^{1}$, Cosimo Palazzo ${ }^{1}$, Giovanni Aloisio ${ }^{1}$, Sandro Fiore ${ }^{1}$, Antonio Bonaduce ${ }^{1}$, Yogesh Vittal Kumkar ${ }^{1}$, Stefania Angela Ciliberti ${ }^{1}$, Ivan Federico ${ }^{1}$, Gianandrea Mannarini ${ }^{1}$, Paola Agostini ${ }^{1}$, Roberto Bonarelli ${ }^{1}$, Sara Martinelli ${ }^{1}$, Giorgia Verri ${ }^{1}$, Letizia Lusito $^{1}$, Davide Rollo $^{2}$, Arturo Cavallo ${ }^{2}$, Antonio Tumolo ${ }^{2}$, Tony Monacizzo ${ }^{2}$, Marco Spagnulo $^{2}$, Rorberto Sorgente ${ }^{4}$, Andrea Cucco ${ }^{4}$, Giovanni Quattrocchi ${ }^{4}$, Marina Tonani ${ }^{5}$, Massimiliano Drudi ${ }^{6}$, Paola Nassisi ${ }^{1}$, Laura Conte $^{1}$, Laura Panzera ${ }^{1}$, Antonio Navarra ${ }^{1}$, and Giancarlo Negro ${ }^{2}$

${ }^{1}$ CMCC, Fondazione Centro Euro-Mediterraneo sui Cambiamenti Climatici, via Augusto Imperatore 16, 73100 Lecce, Italy

${ }^{2}$ Links S.p.A., Links Management and Technology, via R. Scotellaro, 73100 Lecce, Italy

${ }^{3}$ Università di Bologna, viale Berti-Pichat, 40126 Bologna, Italy

${ }^{4}$ CNR-IAMC, Consiglio Nazionale delle Ricerche - Istituto per l'Ambiente Marino Costiero, Oristano, Italy

${ }^{5}$ INGV, Istituto Nazionale di Geofisica e Vulcanologia, Via Donato Creti 12, 40128 Bologna, Italy now at Met Office, FitzRoy Road, Exeter, UK

${ }^{6}$ INGV, Istituto Nazionale di Geofisica e Vulcanologia, Via Donato Creti 12, 40128 Bologna, Italy

Correspondence to: Giovanni Coppini (giovanni.coppini@cmcc.it)

Received: 15 May 2016 - Discussion started: 21 June 2016

Accepted: 22 December 2016 - Published: 13 April 2017

\begin{abstract}
Reliable and timely information on the environmental conditions at sea is key to the safety of professional and recreational users as well as to the optimal execution of their activities. The possibility of users obtaining environmental information in due time and with adequate accuracy in the marine and coastal environment is defined as sea situational awareness (SSA). Without adequate information on the environmental meteorological and oceanographic conditions, users have a limited capacity to respond, which has led to loss of lives and to large environmental disasters with enormous consequent damage to the economy, society and ecosystems. Within the framework of the TESSA project, new SSA services for the Mediterranean Sea have been developed. In this paper we present SeaConditions, which is a web and mobile application for the provision of meteorological and oceanographic observation and forecasting products.

Model forecasts and satellite products from operational services, such as ECMWF and CMEMS, can be visualized in SeaConditions. In addition, layers of information related to bathymetry, sea level and ocean-colour data (chl $a$ and water transparency) are displayed. Ocean forecasts at high
\end{abstract}

spatial resolutions are included in the version of SeaConditions presented here.

SeaConditions provides a user-friendly experience with a fluid zoom capability, facilitating the appropriate display of data with different levels of detail. SeaConditions is a single point of access to interactive maps from different geophysical fields, providing high-quality information based on advanced oceanographic models.

The SeaConditions services are available through both web and mobile applications. The web application is available at www.sea-conditions.com and is accessible and compatible with present-day browsers. Interoperability with GIS software is implemented. User feedback has been collected and taken into account in order to improve the service. The SeaConditions iOS and Android apps have been downloaded by more than 105000 users to date (May 2016), and more than 100000 users have visited the web version. 


\section{Introduction}

Sea situational awareness (SSA) in terms of the adequate dissemination of marine environmental data to users and stakeholders is strategically important for the management and safety of the Mediterranean Sea and its coastal areas. When there is little or no adequate information the response capacity for operations at sea is consequently reduced, leading to a potential loss of lives and socio-economic damage. SSA is being addressed by TESSA (Development of TEchnology for Sea Situational Awareness), an industrial research project funded under the PON (Programma Operativo Nazionale) "Ricerca and Competitività 2007-2013" programme of the Italian Ministry for Education, University and Research.

The first end-user-oriented service to be developed in TESSA was SeaConditions which was presented in its preliminary version in Lecci et al. (2015). The present paper describes the final version of SeaConditions with a comprehensive overview of all the components and of the different web and mobile applications. SeaConditions is an open service providing ocean and weather forecasts, remote sensing data and bathymetry for the Mediterranean Sea, both for PCbased and mobile channels. At the end of the TESSA project, the SeaConditions began to be marketed as a commercial application in line with the industrial development of the funding framework aimed at increasing the competitiveness of the marine/maritime sector(s).

SeaConditions provides ocean forecasts to a wide community of diverse users with different requirements. The high level of customization possible with the MySeaConditions component is a response to the very specific needs of some of the users, such as repeated bulletins in a specific location and only for selected variables (e.g. waves, wind, water temperature).

Other software or web portals similar to SeaConditions such as Weather4D and Meteomed do not offer the same integration with marine data. Meteomed does not offer currents and high-resolution model data. Neither Weather4D nor Meteomed offer the zoom capacity in the free version or bathymetry as one of the products.

Since the beginning of the Mediterranean Forecasting System (MFS), INGV (National Institute of Geophysics and Volcanology) has developed web interfaces to visualize the oceanographic products of the Mediterranean Sea (http://medforecast.bo.ingv.it/mfs-copernicus) developed in the different projects (MFSPP, MFSTEP, MYOCEANs and now CMEMS $^{1}$ ). The INGV website is more science-oriented with respect to SeaConditions and consists of static images without any zoom capability and without providing meteorological forecasts. Many other systems (e.g. Puertos forecasting system portus.puertos.es; CYCOFOS forecasting systems http://www.

\footnotetext{
${ }^{1}$ Copernicus Marine Environment Monitoring Service http:// marine.copernicus.eu
}

oceanography.ucy.ac.cy/cycofos/high-resolution.html), most of which are part of MONGOOS (http://www.mongoos.eu/ in-situ-and-forecasts), provide access to the visualization services of oceanographic forecasting products. However all of them apply to subregional areas only and not for the entire Mediterranean sea, and most of them have a limited zoom capability, without providing access to atmospheric data. Only a few of these systems provide access through mobile applications and only for subregional areas of the Mediterranean Sea (IMAR by Puertos available on Google store for the western Mediterranean Sea).

SeaConditions was designed using a user-centred approach. Through several technical meetings and large workshops with tens of users, we analysed users' requirements, co-designed the application and co-developed some of the features such as the visualization of the variable palette and colours to be used, testing the different versions and collecting feedback. In the field of weather services support, SeaConditions brings together four different types of SSA information: (1) meteorological forecasts, (2) state of the art marine forecasts, (3) remote sensing observations and (4) bathymetry.

The paper is organized as follows. In Sect. 2 the operational chain is introduced and subdivided into the operational centres and SSA Platform. Section 3 presents the web portal, the mobile applications and the customizable version web application of MySeaConditions. Section 4 provides an overview of the interactions with testers and end users. Section 5 presents concluding remarks and suggestions for future directions.

\section{The operational chain}

Figure 1 shows the SeaConditions operational chain comprising five main steps:

1. data acquisition of the generic forecast products from CMEMS, from INGV for the oceanographic forecasts and from CNMCA (Centro Nazionale di Meteorologia e Climatologia Aeronautica) of the Italian Met-Office (Aeronautica Militare) for the meteorological forecasts;

2. the production of subregional model forecasts, available for the Adriatic Sea Forecasting System (AFS) produced by CMCC and the Tyrrhenian and Sicily Channel Forecasting System (TSCFS) produced by CNR-IAMC;

3. post-processing by the CMCC of the products provided by CMEMS, AFS and TSCFS;

4. map rendering by Links S.p.A. performed on the dedicated SSA platform generating information (e.g. maps and graphs) to the end-user. The data are presented as services in standard formats (such as REST, TMS, WMTS) for integration into end-user applications. This activity is conducted by Links S.p.A. 
5. Finally, the service is provided to different end-user applications in a multi-channel mode (web and mobile, such as tablet and smartphone). This activity is conducted by Links S.p.A.

The following subsections describe the components of the SeaConditions production and delivery chains mentioned above.

\subsection{The operational centres (input data, subregional models and post-processing)}

The SeaConditions operational chain starts every day with the downloading of products provided by CMEMS, CMCC and CNR-IAMC. CMCC perform the downloading and postprocessing of the products which are then transferred to Links S.p.A (a small-medium enterprise).

The IFS is the numerical model producing atmospheric data with a $1 / 8^{\circ} \times 1 / 8^{\circ}$ horizontal resolution and is run and delivered by ECMWF. The Italian National Meteorological Centre (CNMCA) access the IFS data produced by ECMWF and makes them available to INGV. The IFS products used in SeaConditions consist of 5-day forecasts with a temporal resolution of $3 \mathrm{~h}$ for the first 3 days and $6 \mathrm{~h}$ for the remaining 2 days. The variables used in SeaConditions are temperature at $2 \mathrm{~m}$, total cloud cover, winds at $10 \mathrm{~m}$, mean sea level pressure and total precipitation. CMCC and CNR-IAMC download IFS data to run their respective oceanographic models, and CMCC also includes them in SeaConditions as atmospheric products. The main characteristics of the forecasting systems used by SeaConditions are presented in Table 1.

The oceanographic model products included in SeaConditions are the following:

- CMESM MED-MFC products for ocean surface currents and temperature for the Mediterranean Sea in the framework of CMEMS;

- MFS/INGV data on waves for the Mediterranean Sea;

- AFS data, for the ocean surface currents and temperature for the Adriatic Sea as produced by CMCC;

- TSCFS data, for the ocean surface currents and temperature for the Tyrrhenian Sea and the Sicily Channel.

The CMESM MED-MFC Mediterranean Forecasting System (MFS) is a coupled hydrodynamic-wave forecasting system developed and operated by INGV from 2000 and now delivered in the framework of the CMEMS Med-MFC. The hydrodynamic model is based on the Nucleus for European Modelling of the Ocean-Ocean Parallelise (NEMO-OPA) version 3.4 implemented in the Mediterranean Sea at $1 / 16^{\circ}$ horizontal resolution and 72 unevenly spaced vertical levels (Oddo et al., 2009; Tonani et al., 2009). The wave dynamic is based on the WaveWatch-III (WW3) model (Tolman, 2009) implemented for the Mediterranean Sea. The model is forced at the surface by atmospheric data on analyses and forecasts produced by the ECMWF with a frequency of $6 \mathrm{~h}$ and a spatial resolution of $0.25^{\circ}$ (Tonani et al., 2008). The solutions are then corrected by the variational assimilation (based on a 3-D-VAR scheme) of temperature and salinity vertical profiles and along-track satellite sea level anomaly observations (Dobricic and Pinardi, 2008). MFS produces a 10-day forecast initialized by a simulation every day except on Tuesdays, when the analysis is used instead of the simulation. The products are composed of 3-D hourly and daily mean fields of potential temperature, salinity, zonal and meridional velocity, and by 2-D hourly and daily mean fields of sea surface height and wave parameters, such as the significant wave height, direction and mean period.

AFS is a hydrodynamic forecasting system which was developed by INGV in April 2003. Since July 2013 it has been operated by the CMCC in Lecce (Italy). The hydrodynamic model is based on the Princeton Ocean Model (POM) implemented in the Adriatic Sea at a $1 / 45^{\circ}$ horizontal resolution and 31 sigma vertical levels (Oddo et al., 2005, 2006; Guarnieri et al., 2010). The domain encompasses the whole Adriatic basin and extends south of the Otranto channel into the northern Ionian Sea. The model is forced at the surface by atmospheric data on analyses and forecasts produced by ECMWF with a frequency of $6 \mathrm{~h}$ and a spatial resolution of $0.25^{\circ}$. The river input into the basin has been implemented through river climatology (Raicich, 1994) for all the rivers except for the Po, which is a very important forcing for the Adriatic Sea (Oddo et al., 2005, 2006; Guarnieri et al., 2010). The Po run-off is implemented on a daily basis. The initial and lateral boundary conditions for temperature, salinity and velocity come from MFS (Pinardi et al., 2003; Tonani et al., 2008). In order to have the best estimate of the state of the sea for a new forecast, the daily cycle of the AFS system is combined once a week with a weekly cycle every Wednesday and provides the best initial conditions for the forecast. Every day, AFS produces a 9-day forecast and the products are composed of 3-D, hourly and daily mean fields of potential temperature, salinity, zonal and meridional velocity, and by 2-D hourly and daily mean fields of sea surface height.

TSCFS is the evolution and extension of the Sicily Channel Regional Model (SCRM, Sorgente et al., 2003, 2011; Olita et al., 2007, 2012), which has produced operational forecasts since 2003. It represents a new implementation of the Princeton Ocean Model at a $1 / 48^{\circ}$ of horizontal resolution and 31 vertical levels and provides a daily 5-day forecast of the 3-D ocean hydrology (temperature, salinity) and circulation in the central Mediterranean basin. Forecast fields are produced in "slave" mode, which means that they are reinitialized every day from the coarse model (MFS, Tonani et al., 2009), which also provides the boundaries for the system. At the surface, it receives $6 \mathrm{~h}$ forcing fields from ECMWF. Momentum and heat fluxes are computed interactively by the model through bulk formulae specific to the Mediterranean (Bignami et al., 1995; Castellari et al., 1998). Since Jan- 


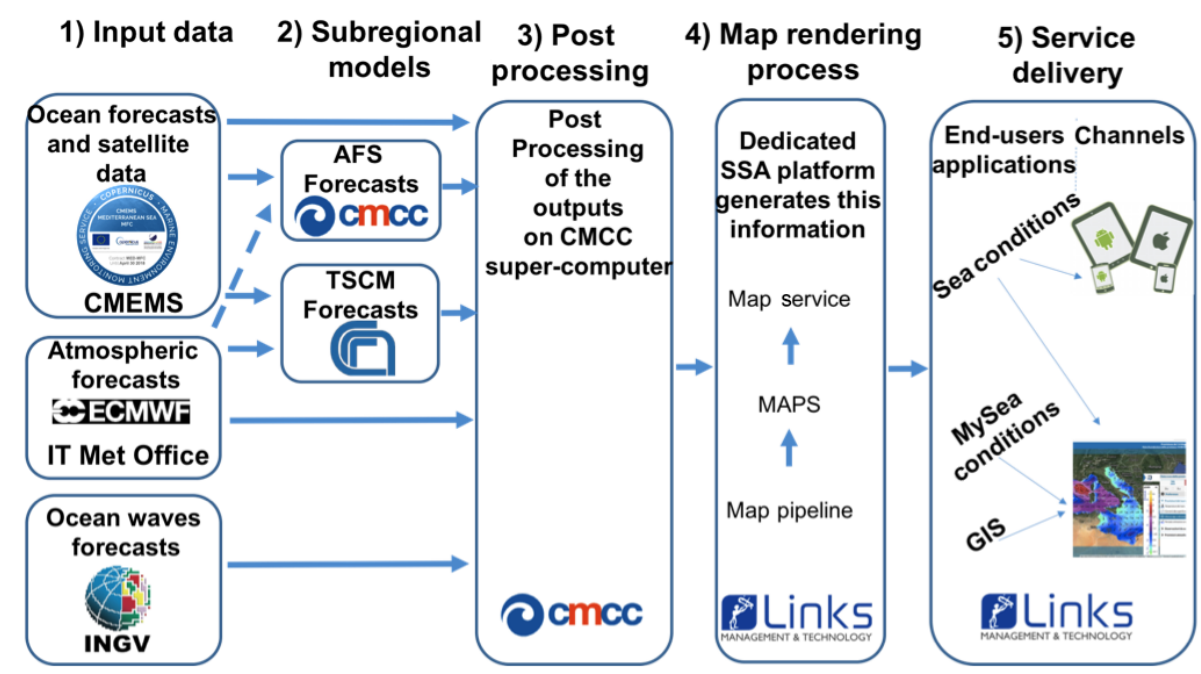

Figure 1. The SeaConditions operational chain: the external data providers ECMWF, CMEMS and INGV feed the data production and collection centres, where high spatial resolution ocean forecast models are run. The output fields are then sent to the SSA platform for map rendering before they are made available to the end-user applications, through both web and mobile channels.

Table 1. Main characteristics of the forecasting systems used by SeaConditions.

\begin{tabular}{|c|c|c|}
\hline Forecasting system name & Model name & Main characteristics \\
\hline CMESM MED-MFC (MFS) & NEMO-OPA version 3.4 & $\begin{array}{l}\text { Horizontal resolution: } 1 / 16^{\circ} \\
\text { Vertical levels: } 72 \\
\text { unevenly spaced }\end{array}$ \\
\hline MFS/INGV waves & WaveWatch-III (WW3) & Horizontal resolution: $1 / 16^{\circ}$ \\
\hline IFS & $\mathrm{IFS}^{2}$ & Horizontal resolution $1 / 4^{\circ}$ \\
\hline AFS & Princeton Ocean Model (POM) & $\begin{array}{l}\text { Horizontal resolution: } 1 / 45^{\circ} \\
\text { Vertical levels: } 31 \sigma \\
\text { vertical levels }\end{array}$ \\
\hline TSCFS & Princeton Ocean Model (POM) & $\begin{array}{l}\text { Horizontal resolution: } 1 / 48^{\circ} \\
\text { Vertical levels: } 31 \sigma \\
\text { vertical levels }\end{array}$ \\
\hline
\end{tabular}

uary 2015, the system has also produced operational analyses through a 3-D-variational data assimilation (DA) module. The DA software assimilates along-track sea level anomalies on a daily basis (Dobricic et al., 2007; Olita et al., 2012).

The above-mentioned products delivered by the oceanographic and atmospheric numerical models are automatically downloaded every day by providers and stored in the CMCC supercomputer cluster as soon as they are available on the web servers of the providers. In the case of high-resolution ocean products for the Adriatic Sea (AFS by CMCC), the data are directly available on the cluster.

The first version of the operational chain for SeaConditions is described in Lecci et al. (2015), and here we summarize the main steps and highlight the novelties.

1. The scheduling phase dispatches the data to the different phases in order to implement a high parallelism for the computations.
2. The pre-processing phase deals with the time-splitting and conversion to the CF (Climate and Forecast). NetCDF (Network Common Data Form) format of the input data. At this stage, the operational chain subsamples the variables of interest for the requested temporal frames and for the surface level.

3. The statistic phase computes statistics such as minimum, maximum, mean and standard deviation over the input data in order to plot the appropriate palette in the rendering phase.

4. The interpolation phase is a demanding phase from a resource point of view which (at the end) delivers high spatial resolution data $(150 \mathrm{~m})$. Atmospheric fields are provided over the original $1 / 4^{\circ}$ resolution (about $25 \mathrm{~km}$ ) grid except for $10 \mathrm{~m}$ winds, which are interpolated over a target grid of $2 \mathrm{~km}$ resolution. 
5. In the extrapolation phase, ocean products in the coastal areas are extrapolated towards land using a procedure called SeaOverLand (De Dominicis et al., 2013; Mannarini et al., 2016), which extrapolates the original data, considering an average of the eight nearest values for each cell grid point and then doing different iterations. This procedure optimally fills the gaps that remain between the ocean model domain and the high-resolution coastline. A high-resolution mask is then applied to remove the extrapolated ocean data on land.

6. The renaming and packing phase is where, once transformed, all the data are packed using NetCDF libraries, with a decrease in their dimension to $1 / 10$ of their original size.

The steps listed above are performed through a series of automated processing chains running $24 \mathrm{~h}$ a day, 7 days a week (Lecci et al., 2015).

SeaConditions also provides remote sensing products such as chlorophyll $a$ concentration and the transparency of seawater. These data are retrieved by satellite and are displayed on a $1 \mathrm{~km}$ spatial resolution grid by the CMEMS. Once available online, the data are automatically downloaded on the CMCC supercomputing cluster and transformed into the final output requested by SeaConditions.

In addition to remote sensing and model data, SeaConditions also provides information on the sea level time series combined with 2-day sea level forecasts products for the Italian seas. Sea level observations are delivered (36 stations in May 2015) by the Italian national tide gauge network (Rete Mareografica Nazionale, RMN, of the Istituto Superiore per la Protezione e la Ricerca Ambientale, ISPRA) which are then combined by CMCC with an ensemble forecasting products of sea level.

In addition to the above-mentioned products, SeaConditions provides bathymetric information for the Mediterranean Sea from the Italian Hydrography Institute of the Navy (Istituto Idrografico della Marina Militare, IIM). Bathymetry and rocks awash data are provided by the IIM and were transformed by Links S.p.A. and CMCC in static layers which have been made available on SeaConditions ${ }^{3}$.

\subsection{The SSA platform}

The SSA platform (Marra et al., 2016; Lecci et al., 2015) collects, transforms and provides forecast and observational products as information across a variety of mechanisms, such as web and mobile applications.

The following are the core platform functional blocks: (1) the computing grid provides the platform with a highly scalable computing capability for processing forecast data and producing ready-to-use information (e.g. map rendering); (2) the rendering pipeline arranges and supervises batch

\footnotetext{
${ }^{3}$ Since the end of the TESSA project, information on sea level and bathymetry are no longer supported.
}

rendering jobs for processing NetCDF data; (3) the forecast service provides Application Programming Interfaces (APIs) for consuming the published forecast information (INSPIRE compliant), both as tiled maps and related metadata. Finally, it provides the on-demand rendering functionality which permits on-the-fly single-tile rendering, caching results on a local storage device for later retrieval. This improves global performances while keeping the actual resource usage (e.g. disk space for storing maps) at the required minimum amount (Lecci et al., 2015).

\section{The application}

\subsection{The portal}

SeaConditions is aimed at the general public who wish to know the meteorological and oceanographic conditions at sea. It provides a unique point of access to oceanographic and weather forecasts for the entire Mediterranean Sea. The strength of SeaConditions is in integrating and providing high-quality meteorological and oceanographic forecast data with an easy-to-use approach for a large number of consumers.

The first version of SeaConditions was released in July 2012 (Lecci et al., 2015) as a web portal (http://www. sea-conditions.com).

The version at the end of the TESSA project (May 2015) provided the following data:

- forecasting products including

(a) the ocean products of surface temperature and currents, significant wave height and direction and wave period and direction;

(b) the atmospheric products of air temperature at $2 \mathrm{~m}$ a.g.l., mean sea level pressure, total precipitation, total cloud coverage and winds at $10 \mathrm{~m}$ a.g.l.

Once a day, a new set of forecasts for a time span of 4.5 days are published;

- observation and forecast of sea level: observations are related to the past eight days and forecasts for the following two days, which are updated and published once a day;

- sea satellite observations of chlorophyll $a$ concentration, water transparency, updated and published once a day;

- bathymetry and awash rocks. Static layers.

The SeaConditions web portal interface is mainly composed of a map of the Mediterranean Sea and a menu for selecting and displaying data, forecasting time steps and setting display options. The map takes up the main part of the 


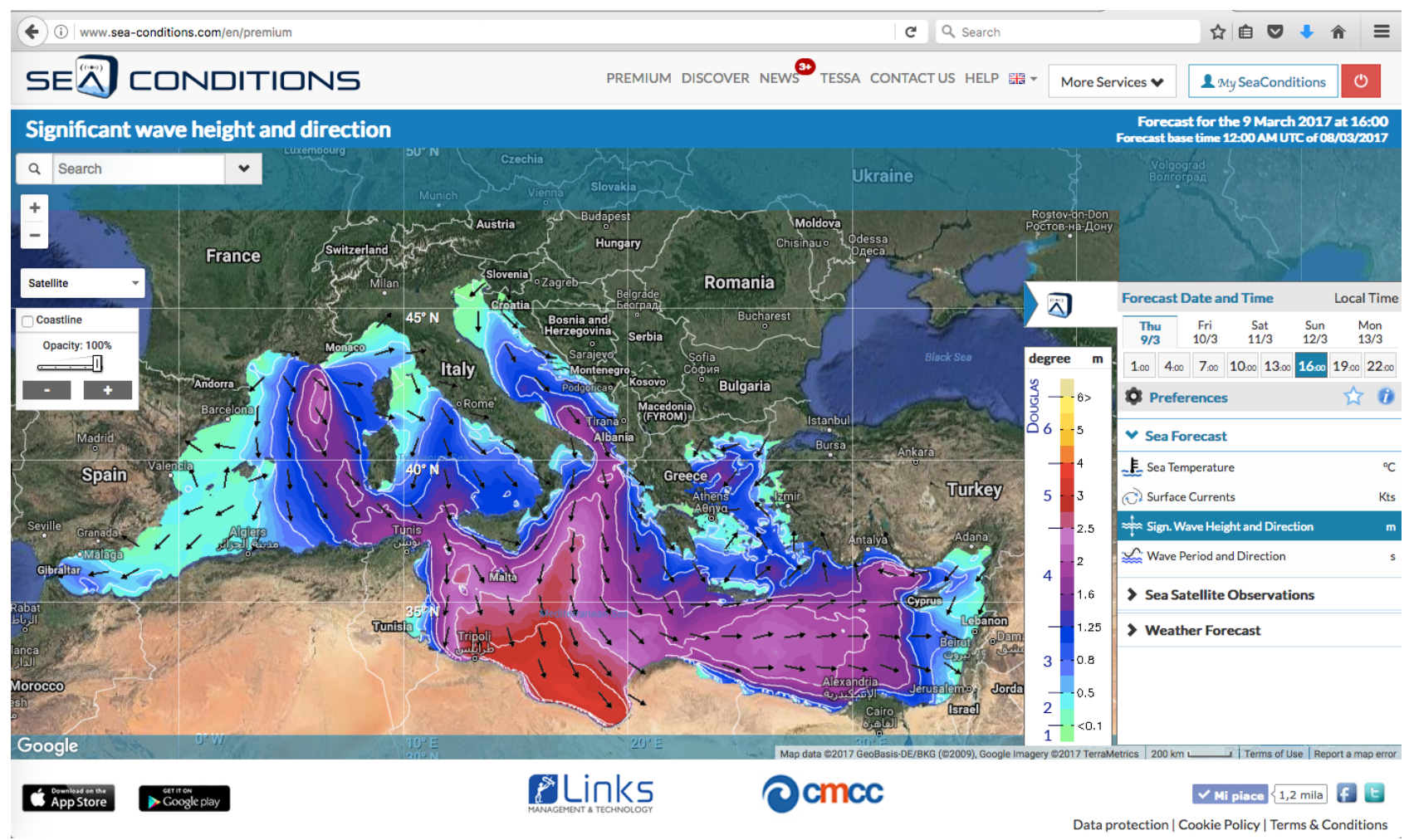

Figure 2. SeaConditions web application showing a significant wave height and direction map.

screen, which enables users to easily browse the forecast information directly on the map.

Forecasts and data are displayed as an information layer superimposed onto a Google map. Different kinds of rendering have been studied and implemented in order to deal with each kind of data: graphical rendering includes map colour shading and/or arrows and graphs representing the forecast trend.

Data expressed only by magnitude are rendered as shaded colour maps, while data expressed by magnitude and direction are displayed by both a shaded layer (for magnitude) and a vector layer in overlay (for direction). Figure 2 shows an example of a map for the significant wave height and direction forecast data.

Different arrows are used by the SSA platform depending on the specific data required. Wave directions are shown as simple arrows, wind directions are displayed as arrows with barbs by also embedding information on wind magnitude. Streamlines have been used to better represent the circulation of currents.

All the forecast data are associated with a reference scale displayed as a legend on the right-hand side of the map. Depending on the kind of data considered, reference scales can be dynamic or static. Dynamic scales are defined every day by calculating the minimum and maximum levels for the Mediterranean Sea, i.e. sea temperature, surface currents, wave period and air temperature. The other refer- ence scales are fixed, based on state-of-the-art knowledge, i.e. wave height (Douglas scale), chlorophyll $a$, water transparency, wind intensity (Beaufort scale), mean sea level pressure, precipitation and cloud cover.

The zoom allows users to inspect specific areas through detailed maps. Figure 3 presents an example of maximum zoom levels obtained for wave heights and directions (Fig. 3a), ocean surface temperature (Fig. 3b), wind (Fig. 3c) and surface ocean currents (Fig. 3d). Ocean surface currents do not obtain the same level of zoom as other variables due to technical limitations in the plotting of curvilinear vectors of streamlines. CMCC and LINKS are working to overcome this problem.

Sea level data are not displayed on a map, but as a set of points, one for each of the selected 22 tide gauge stations along the Italian coastline. For each station, a threshold value is computed. When the sea level goes over this specific value, the station is highlighted in red. By clicking on an individual station, it is possible to obtain sea level trends (both observations and forecasts) by means of a graph (Fig. 4).

In addition to maps (Fig. 3), another way to explore the forecast is by selecting one point on the map and by visualizing the trend for the forecast data in that specific point. Figure 5 shows an example of the trend for a surface current forecast for a specific point on the map.

High-resolution model data (ocean surface temperature and currents) from AFS and TSCFS can be displayed on 
(a)

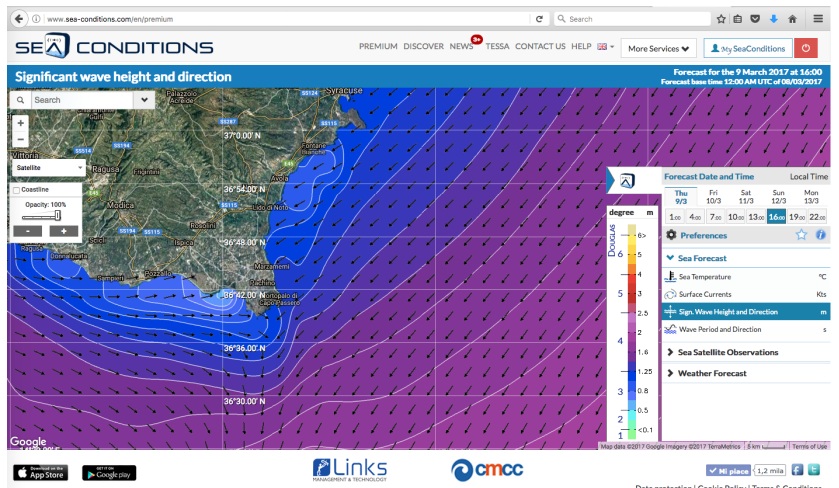

(c)

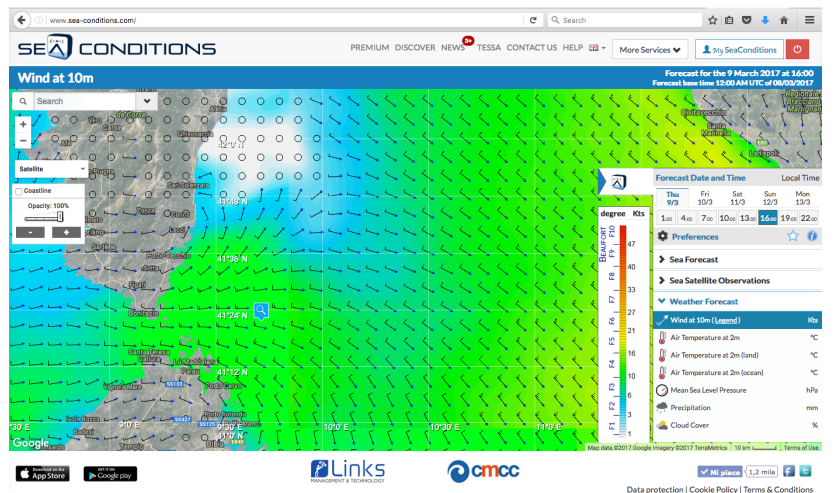

(b)

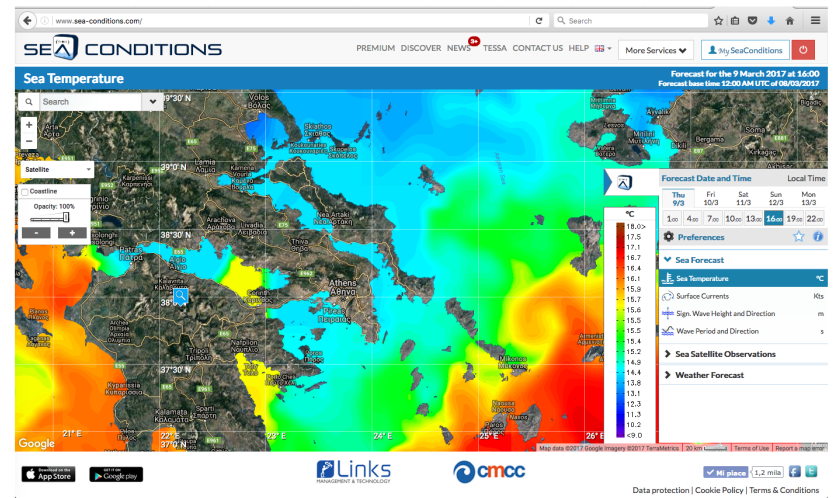

(d)

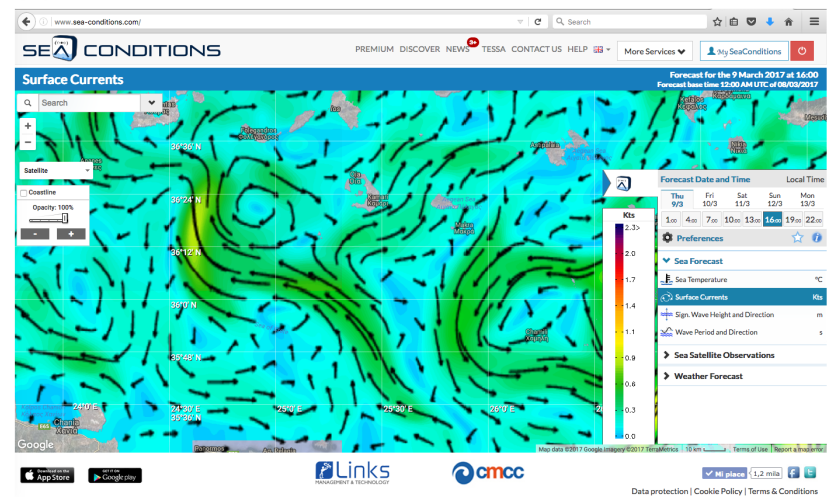

Figure 3. Examples of SeaConditions products at various zoom levels: significant wave height and direction at level 11 (a), surface sea temperature at level 7 (b), wind intensity and direction at level 10 (c) and surface sea currents at level 11 (d).

the web portal. The high-resolution forecasts are displayed only at a medium-high zoom level (after zoom 10 of the Google map) when the user has zoomed in and is starting to explore a subportion of the full domain. The borders of the two areas of the Adriatic Sea and northern Ionian and of the Tyrrhenian Sea and Sicily Channel are highlighted by a dashed white line, so that the users can see the domain of the high-resolution data. Figure 6 illustrates an example of ocean surface currents in the central Mediterranean Sea with the subregional products displayed from the AFS and TSCFS data.

Figure 7 presents the SeaConditions web application feature showing bathymetry (blue line). Data were provided by IIM as a series of maps in a GIS-compatible format. Links S.p.A. and CMCC developed the methodology of merging together the different maps into just one layer for the various Google zoom levels.

Display options (i.e. the default forecast map, unit of measurement) can be set as "once for all" by saving preferences (Lecci et al., 2015).

\subsection{The application for mobile devices}

SeaConditions is a multi-channel and multi-device service available both on web and mobile applications (Lecci et al., 2015) developed for smartphone and tablet, both for Android $^{4}$ and for $\operatorname{IOS}^{5}$ devices.

The SeaConditions mobile app was built on a different paradigm but ensuring the same level of services as the web application. The mobile app was designed by adopting a location-based pattern, thus, in addition to the map views (as in the SeaConditions web version), a navigation structure based on location has been implemented. Specific views have therefore been implemented to provide forecasts related to user position ("around me") and to users' favourite places (Lecci et al., 2015).

In the mobile version, information is displayed not only as maps or graphs, but also using icons. A set of icons was thus designed in order to represent all the data relating to the specific scale.

\footnotetext{
${ }^{4}$ https://play.google.com/store/apps/details?id=it.linksmt.tessa. $\mathrm{scm}$

${ }^{5}$ https://itunes.apple.com/it/app/seaconditions/id737693930? $\mathrm{mt}=8$
} 


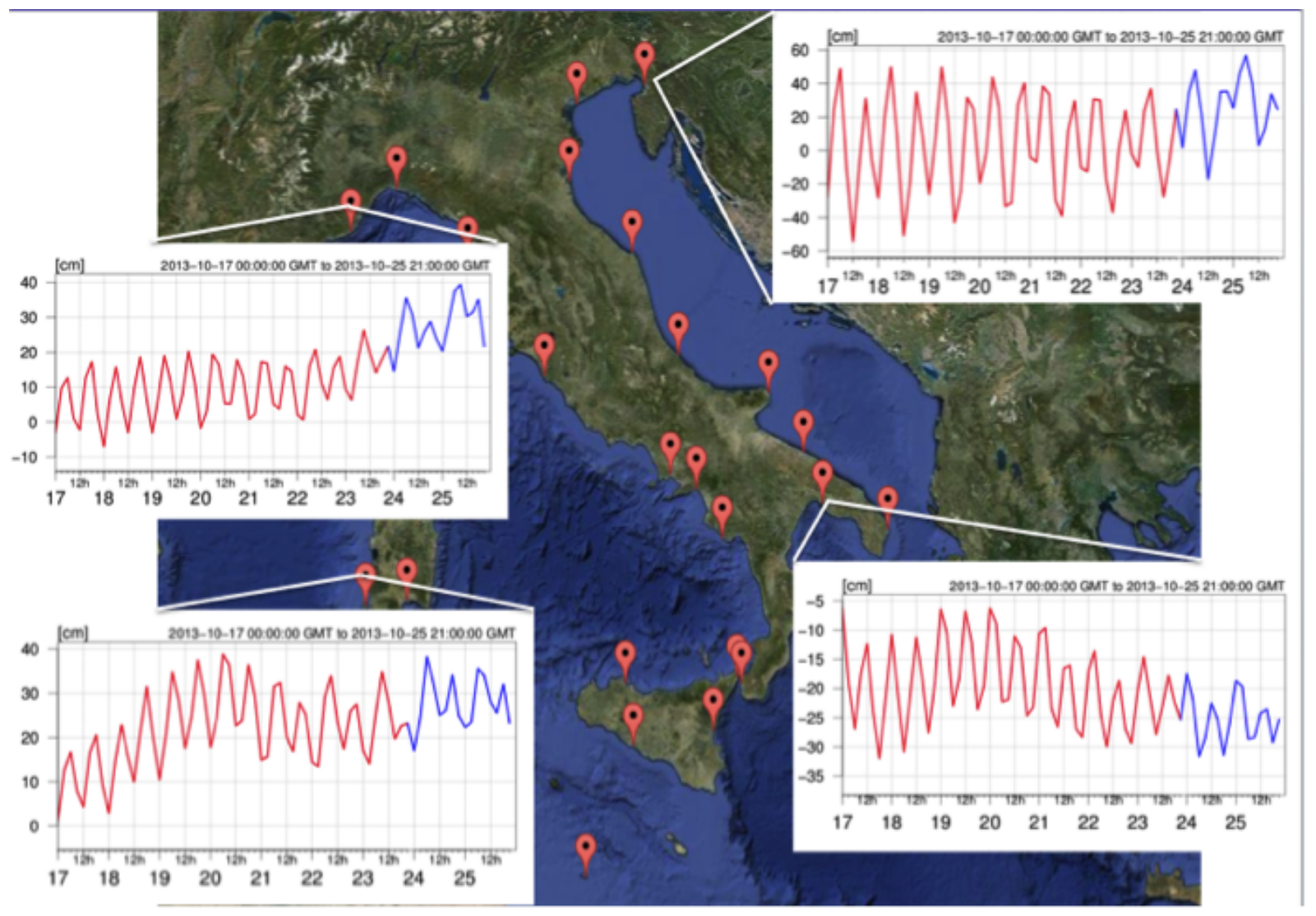

Figure 4. Examples of SeaConditions sea level products in four different locations along the Italian coasts showing the time series of sea level observations in red, and the forecasts for the sea level over the next 2 days in blue.

For the Android version, the main principles were the "material design" guidelines ${ }^{6}$. Material design is "a comprehensive guide for visual, motion and interaction design across platforms and devices". Here are some of the examples of the guidelines that were applied.

- The user interface is organized as sheets of paper in 3-D space. This implies that interfaces have a shadow effect.

- Information is organized into portions of interface ("cards") accessible by a preview/detail pattern. This means that for each card, there are two versions: one with only a subset of information (i.e. only a small image and a caption) and another with the complete set (i.e. the image and complete text).

- Intense colours, bold characters, images and animation are used.

Figure 8 shows two example screens of the Android app. The IOS version was developed in the native IOS language and is customized for iPhones and iPads. Example of the screens from IOS applications are presented in Fig. 9.

Continuous interaction with the users (e.g. workshops, sailing race support, provision of questionnaires) helps to continuously improve SeaConditions in terms of both data and service quality.

\subsection{MySeaConditions web application}

MySeaConditions provides customized user-centred forecasts for any location in the Mediterranean Sea. After registering with SeaConditions, users can access MySeaConditions and benefit from additional services:

- registering favourite locations on the map,

- subscribing to daily email forecast bulletins about favourite locations,

- subscribing to alerts based on customized forecast thresholds for a specific location.

\footnotetext{
${ }^{6}$ http://www.google.com/design/spec/material-design/ introduction.html
} 


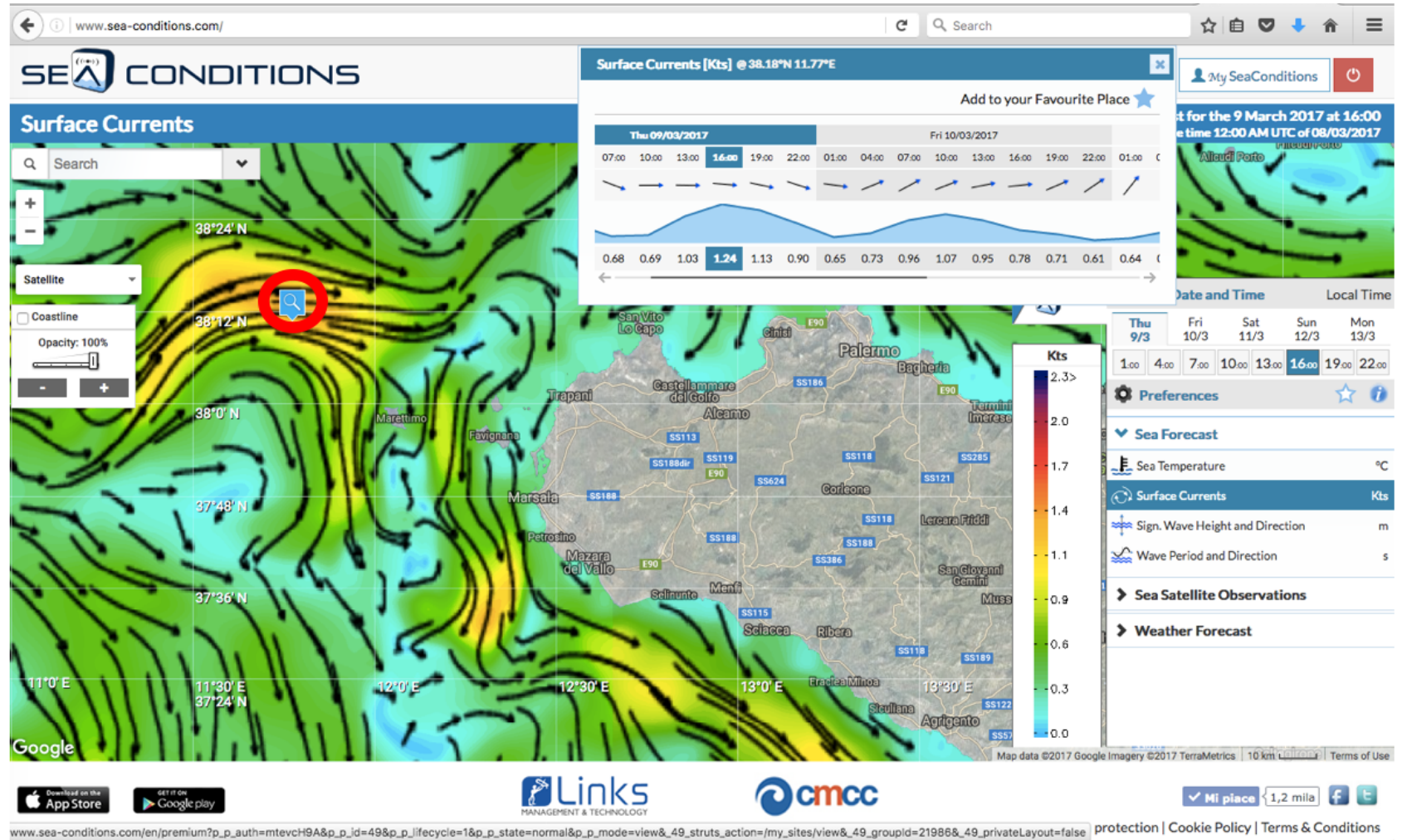

Figure 5. SeaConditions web application - example of surface sea current map and (inset) time series of its intensity and direction variability in relation to the region highlighted with a red circle.

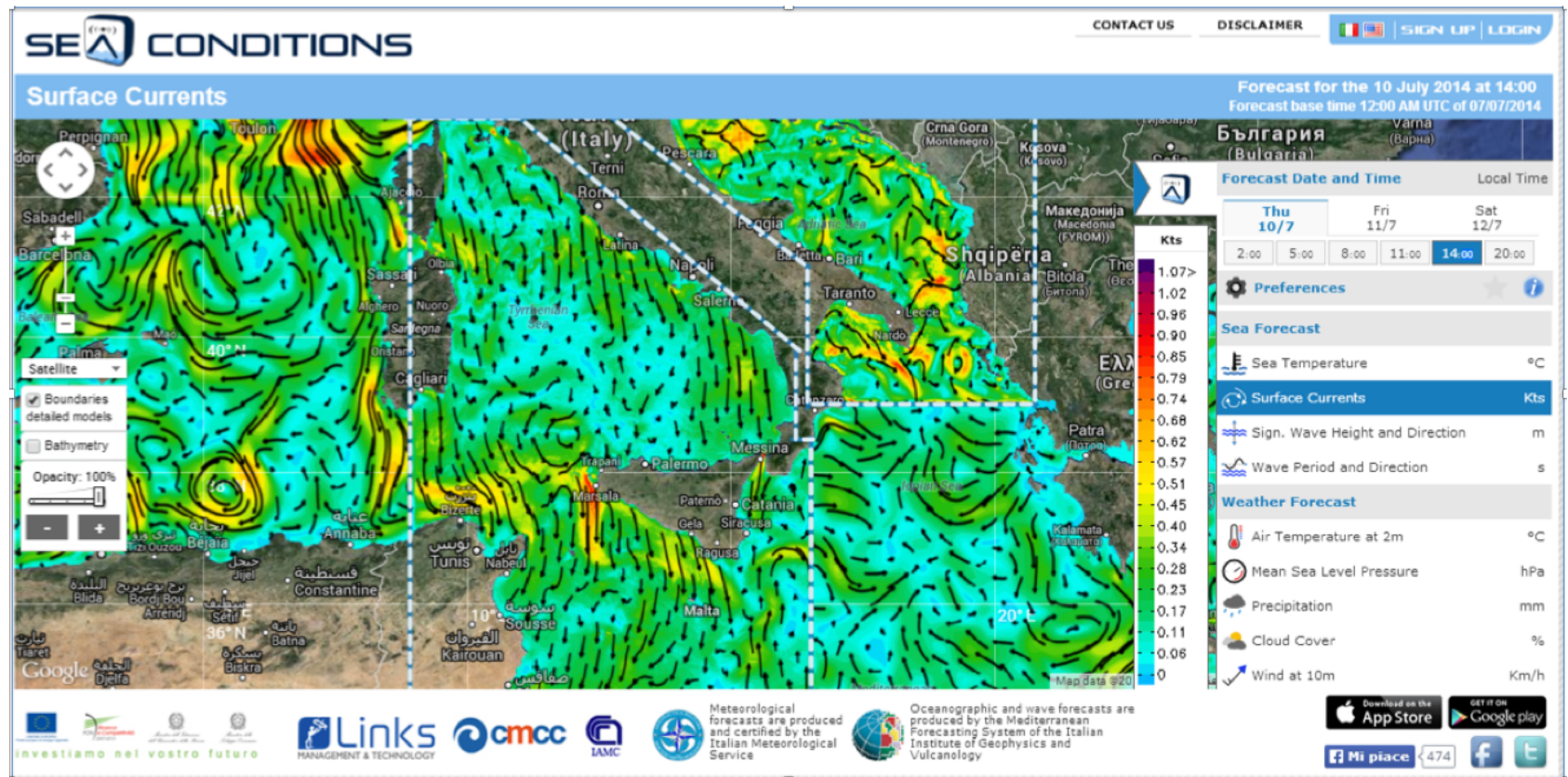

Figure 6. SeaConditions web application - example of ocean surface currents with the subregional products highlighted by a dashed white line. 


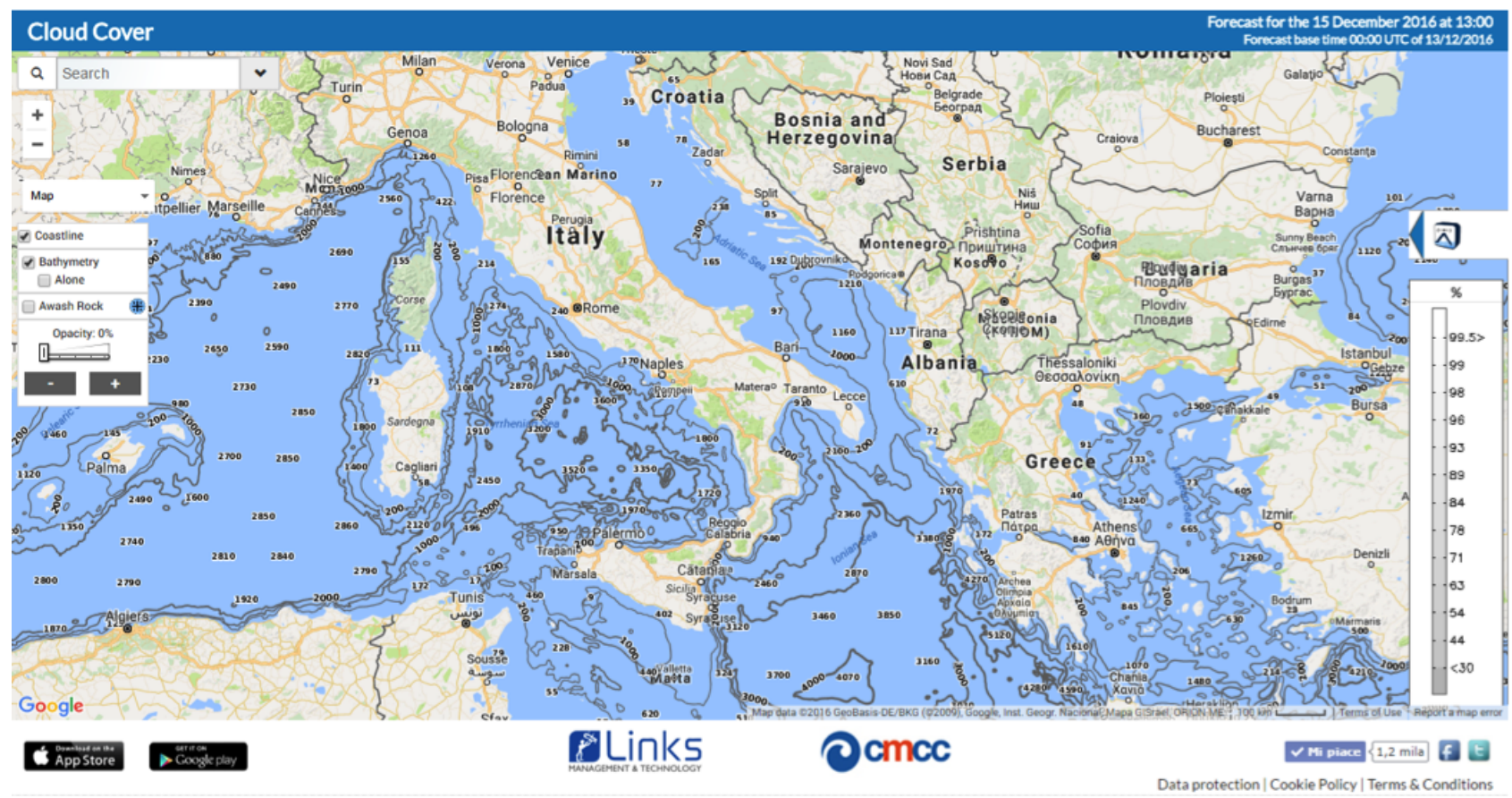

Figure 7. SeaConditions web application - example of bathymetry represented with blue lines.

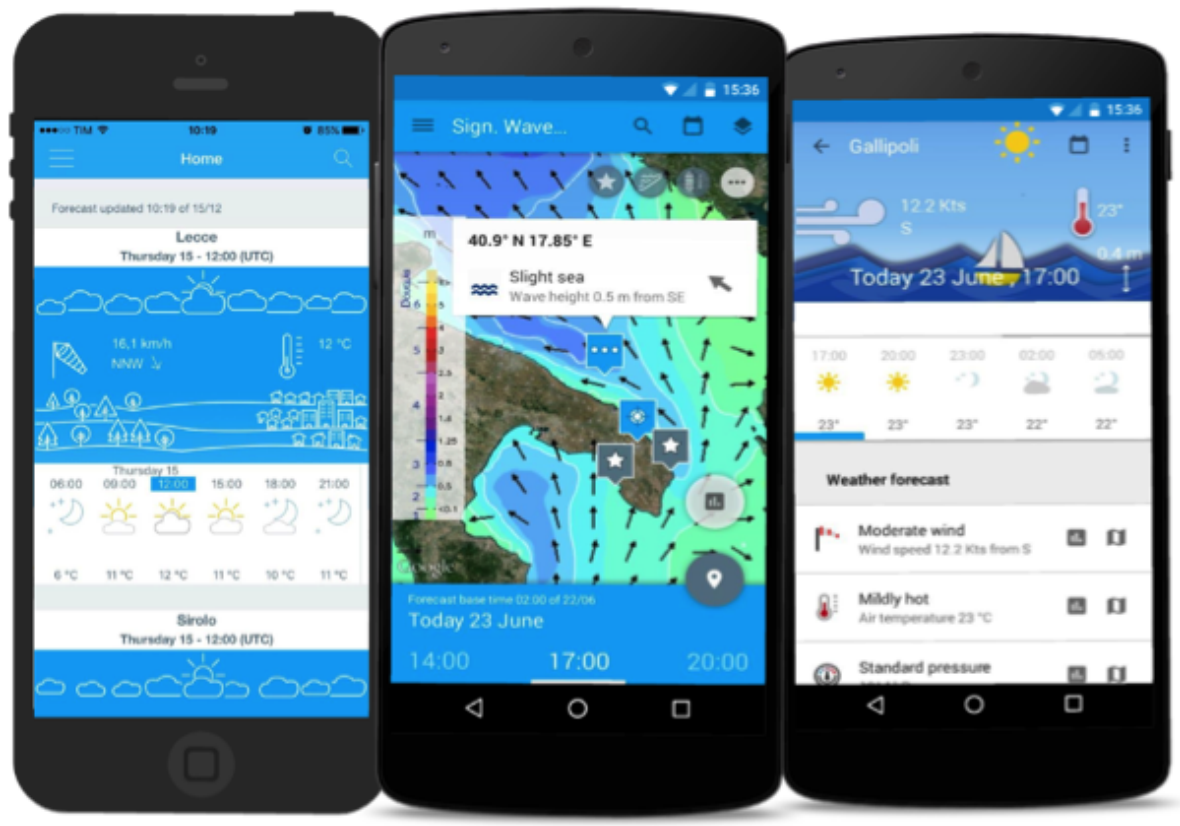

Figure 8. SeaConditions Android mobile app - sample screens.

MySeaConditions (Fig. 10) is only available on the web application of SeaConditions.

\section{Interactions with testers and end users}

SeaConditions provides a service that matches the needs of different users while maintaining easy-to-use and scientifically sound features. Throughout the development of SeaConditions, many stakeholders, ranging from scientists to 
(a)

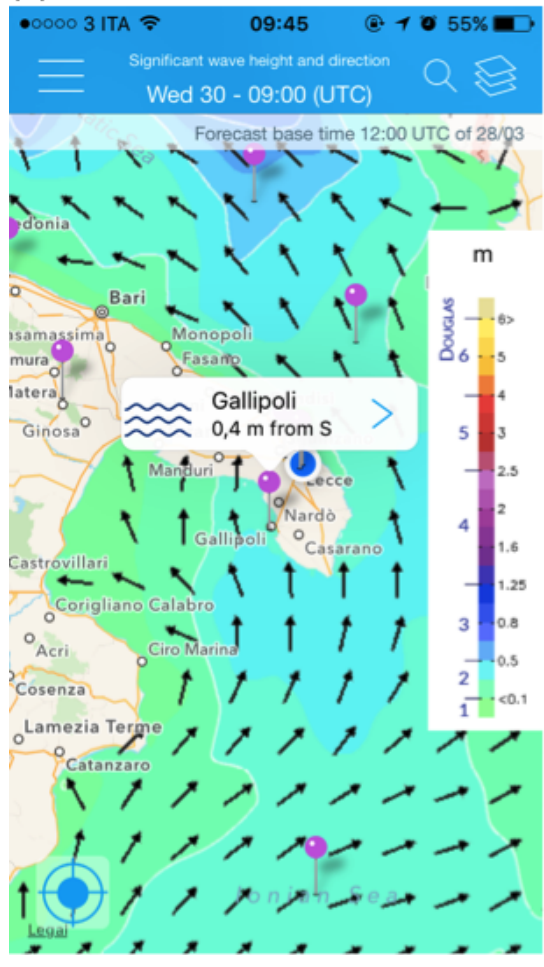

(b)

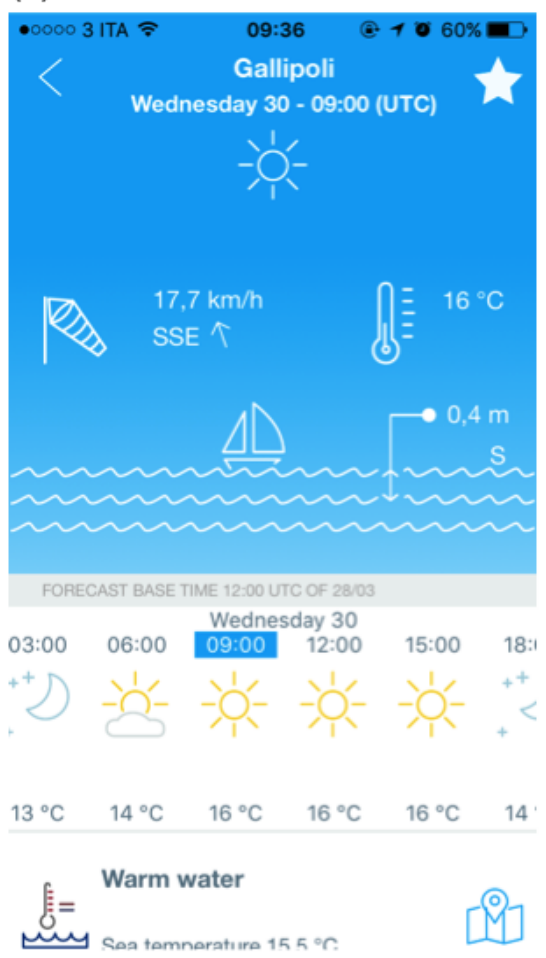

(c)

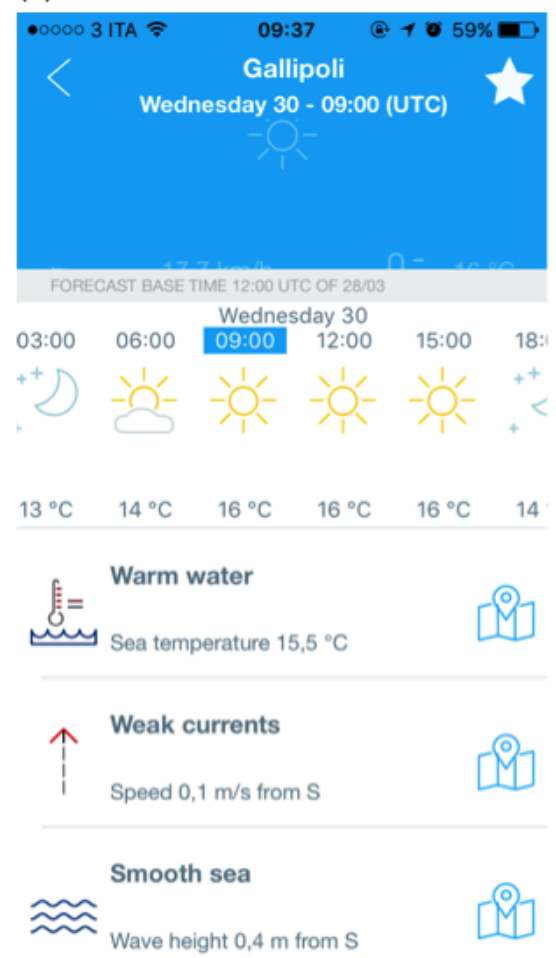

Figure 9. SeaConditions iOS mobile app - sample screens: (a) wave forecast map highlighting a sea location next to Gallipoli $\left(40.05007^{\circ} \mathrm{N}\right.$, $17.96652^{\circ} \mathrm{E}$ ) for which significant wave heights and directions are reported; (b) detailed bulletin for the selected location saved as a favourite place; (c) another view of the bulletin with forecast information on more environmental fields.

public authorities to different practitioners, were involved in testing and giving feedback.

Section 4.1 outlines the general framework that was adopted for users' interactions and Sect. 4.2 presents some interesting results.

\subsection{Framework for the interaction with users}

The methodology adopted to involve users and provide continuous feedback for service improvement is composed of the following main steps:

- identification of the users (e.g. public authorities, such as coast guards, and private users, such as sailors, divers, surfers and fishermen);

- analysis of user requirements, obtained through living labs, participation in conferences/meetings/workshops, personal interactions during dedicated fairs and events;

- experimentation planning, with definition of use cases and scenarios;

- user involvement not only including the experimentation of services in real scenarios, but also a survey of a wider list of users (companies involved in offshore ac- tivities, fishing consortia, marinas, shipping and transport companies);

- collection, categorization and analysis of user feedback.

In our work we applied the living lab methodology to the development of SeaConditions. A living lab is a user-centred, open-innovation ecosystem (Von Hippel, 1986; Chesbrough, 2003; Almirall et al., 2011), integrating concurrent research and innovation processes within a publicprivate-people partnership.

The concept is based on a systematic user co-creation approach integrating research and innovation. These are integrated through the co-creation, exploration, experimentation and evaluation of innovative ideas, scenarios, concepts and related technological artefacts in real-life cases.

An initial set of the service tests was performed with users from academia, which focused on more functional tests on the usability of the user interface (UI), the ability to use the graphical output information, and the performance of the service in terms of zooming, map dragging and dropping, recovery storage output, etc.

Additional non-functional tests regarding UI usability and understanding, and performance in terms of responsiveness and stability when subjected to particular workloads, were submitted to another group of key users, who were also sub- 


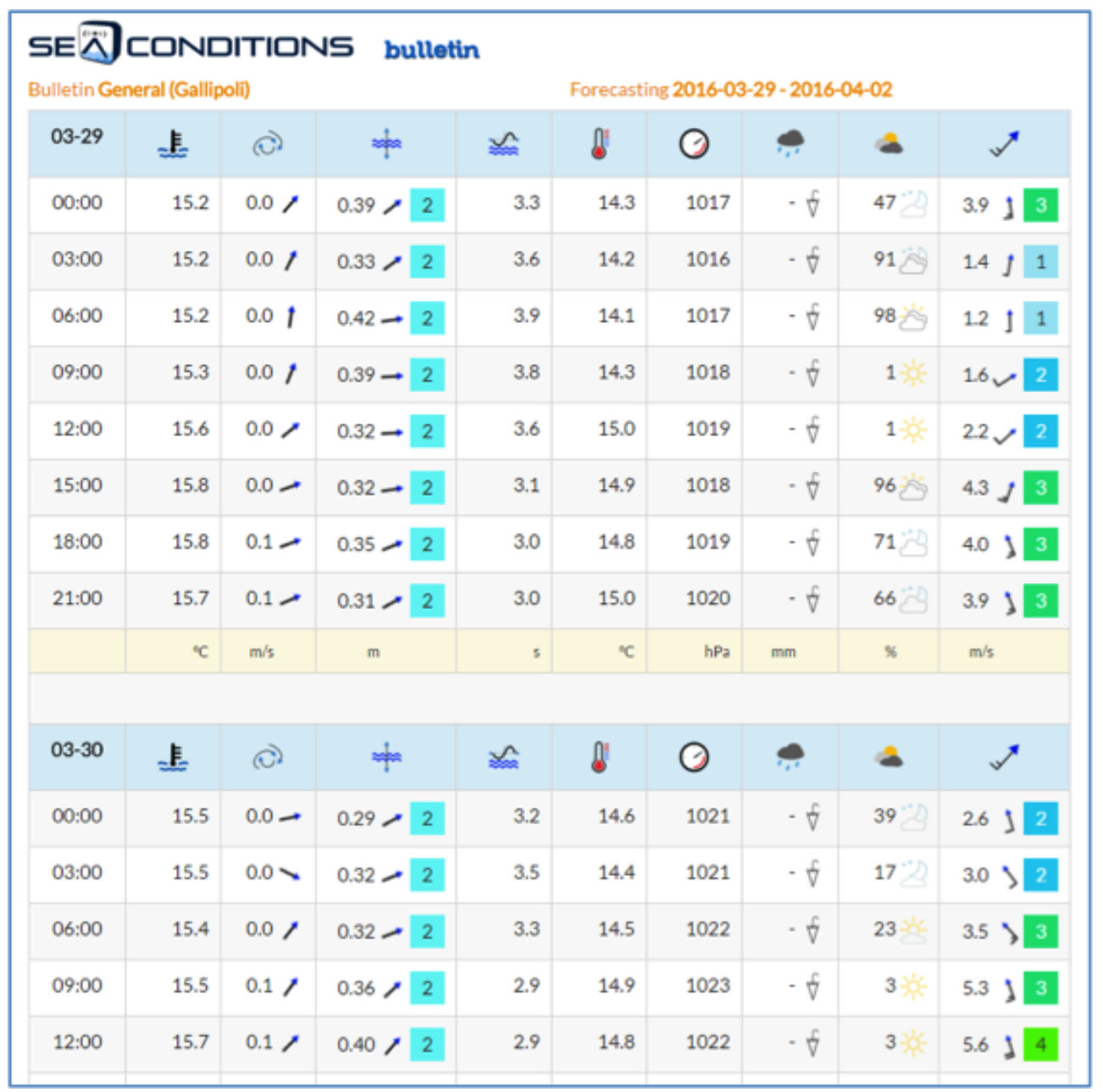

Figure 10. MySeaConditions - example of the daily forecast bulletin generated for the location of Gallipoli in Fig. 8 .

stantially involved in the analysis of users' requirements and corresponding features of SeaConditions. These key users included the Italian Air Force, regional coast guards (Apulia), Regional Agencies for Environmental Protection in Apulia and Emilia Romagna, the Port Authority of Bari, Apulia Region Civil Protection, the sailing association Lega Navale and the Mediterranea sailing project crew.

A wider set of users was involved in the evaluation of the SeaConditions service through an online questionnaire and direct interviews. The questionnaire was composed of two main sections: the first contained questions regarding the knowledge and habits of the users when consulting forecast sites and the readiness to pay for this kind of service. The second section focused on users' interests in the variables provided and on the need for further information and a general evaluation of the usability of SeaConditions. The online questionnaire, developed within the platform SurveyMonkey, was mainly submitted to yacht and sport fishing clubs and surf and diving associations.

\subsection{User results}

A user database with a total number of 456 contacts was collected, including the following categories: offshore activities (62 contacts), fishing (65 contacts), commercial ports (26 contacts), marinas (206 contacts), shipping (ferries and boats) in Italy (55 contacts) and generic transport in Europe, both tourist and commercial (Italy: 4 contacts; Turkey: 2 contacts, France: 5 contacts; Spain: 14 contacts, Greece: 11 contacts; Croatia: 3 contacts; Morocco: 1 contact; Malta: 2 contacts). From these users, around 50 questionnaires were received and Fig. 11 reports various interesting results.

Generally, SeaConditions was well ranked by the respondents, who appreciated the features and level of detail. $70 \%$ of the respondents found the service to be quite good and the remaining $30 \%$ ranked it as an optimal tool. Many respondents reported that they used the system quite often (between once a day and more than once a week) and were also ready to pay for it. However, some suggestions for further improvement of the system were also registered. 

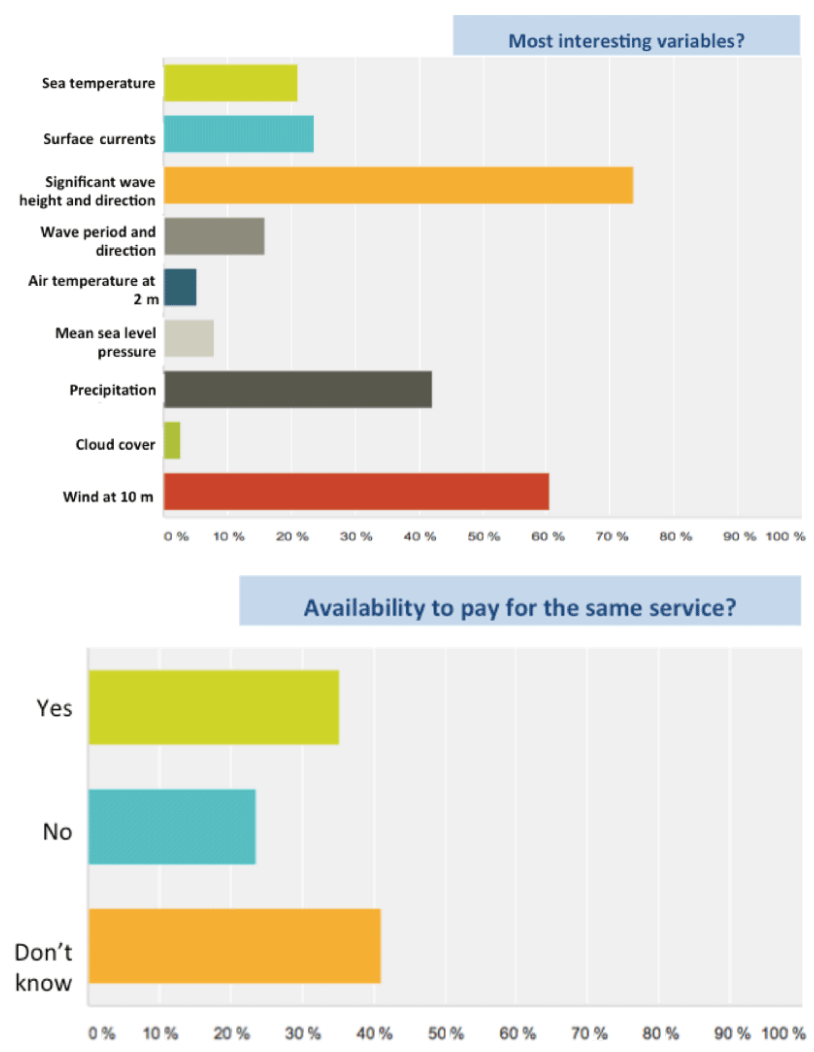
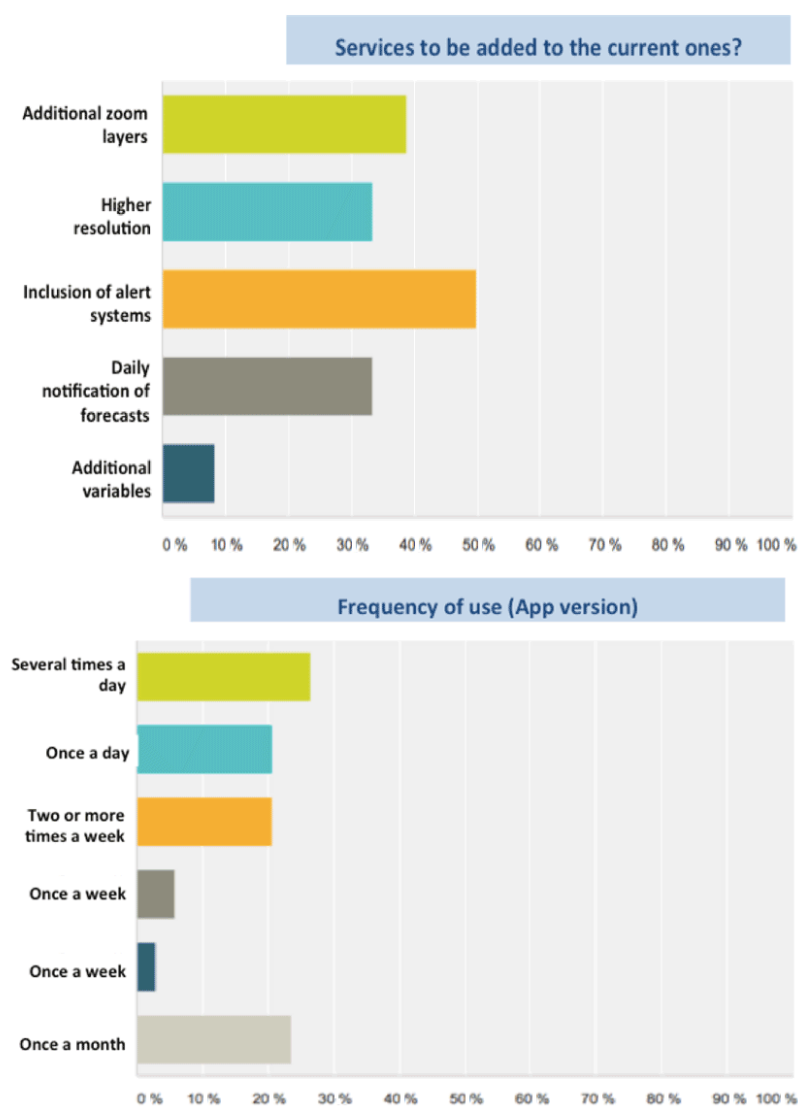

Figure 11. Selected questions and answers by users from SeaConditions online questionnaire.

An example of the interaction with champion users is the Mediterranea project (www.progettomediterranea.com). This 5-year project is a scientific floating laboratory, sailing throughout the Mediterranean Sea, the Black Sea and the northern Red Sea and studying the sea, climate change, water and air pollution, marine biology, currents and anything useful in order to preserve the ecological integrity of the sea. During the navigation in the Mediterranean Sea, the crew used SeaConditions and reported their experience with the service use through visuals and written articles. The videos and articles focused mainly on how the forecasts provided by SeaConditions matched the actual observations of the crew on the sea during the navigation. Feedback from "Mediterranea" is summarized by the following statement from a member of the crew: "A good system, with multiple choices in order to find out the real weather conditions and forecast, easily usable and with a smart design with arrows and colors. A great mix of sources that enables the user to trust the data and the projections. Quite good for sailors due to the comprehensive list of parameters. This is one of the few systems that gives the period of the waves, which is so important in defining the route. In the North Aegean, SeaConditions was a great help in studying the routes and navigating them."

\section{Conclusions and outlook}

SeaConditions represents a new approach to the timely and reliable dissemination of weather-oceanographic forecasting products to users in order to improve their sea situational awareness in the Mediterranean Sea.

SeaConditions is the result of continuous user involvement. Many different champion users were selected to participate in the living lab process, especially in the testing phase, thus providing important feedback for the service improvement. Since the first publication of SeaConditions, more than 100000 people have visited the website and more than 105000 users have downloaded the two mobile apps. User comments are on average very positive, both in terms of service usability and forecast accuracy. A set of users were consulted during the project through dedicated meetings and workshops (living labs) and interviewed through web questionnaires.

The users of SeaConditions are professionals, using the service for their daily working activities (e.g. sailors, fishermen, coast guards, navy personnel), sports enthusiasts (e.g. for sailing, diving, surfing), including those at a professional level, and the public at large, who visit the sea for recreational activities, including tourists. The interaction and di- 
alogue with such a diversified population of users was challenging. Users represented an important investment during the TESSA project and showed that the new technology for SSA is needed in the touristic, recreational and professional activities at sea. The important feedback provided by the users was first used to co-design and co-develop SeaConditions, and then at a later stage to verify and test all the different versions of the SeaConditions portal and apps. Based on user requirements, future activities have been identified, such as the extension to other regions outside of the Mediterranean Sea and an increase in the number of daily updates.

From a scientific and technical point of view, SeaConditions represents an important step forward in the visualization and exploitation of operational oceanography products for SSA. The experience of SeaConditions highlights that research and development should aim to converge towards operational applications as much as possible. This means that the results and findings should be tested in an operational mode and in real-life scenarios by users, which are often situations in which the weak components of the system and bugs are highlighted. Operational testing also helps to demonstrate the effectiveness and importance of research results in supporting societal challenges and in our framework, in highlighting the benefits for maritime safety and for the protection and management of marine environments.

\section{Data availability}

Ocean data used in this paper and used by the operational service (http://www.sea-conditions.com) are produced by CMEMS. The ocean data are available at the CMEMS website http://marine.copernicus.eu and the name of the product is "MEDSEA_ANALYSIS_FORECAST_PHYS_006_001". The atmospheric data used in this paper and used by the operational service are produced by ECMWF. The data for the past can be accessed through a request directly to ECMWF. The atmospheric data are produced by the IFS model operated by the ECMWF. The model outputs are available with 3-hourly resolution for the first 3 days after the analysis, the horizontal resolution is $1 / 8(12.5 \mathrm{~km})$, and the forecasts refer to the 12:00 UTC analysis. Data access can be requested at ECMWF at this link: http://www.ecmwf.int/en/forecasts/ datasets/set-i\#I-i-a.

Competing interests. The authors declare that they have no conflict of interest.

Acknowledgements. This work was performed within the framework of the TESSA project PON01_02823, supported by PON Ricerca \& Competitività 2007-2013 co-founded by the EU (Fondo Europeo di sviluppo regionale), MIUR (Ministero Italiano dell'Università e della Ricerca) and MSE (Ministero dello Sviluppo Economico). We would like to thank Copernicus Marine Envi- ronment Monitoring Service - CMEMS for the ocean forecasting products, Istituto Idrografico Marina Militare for the bathymetry data, Servizio Meteorologico Aeronautica Militare Italiano for the weather forecasting products and Istituto Superiore per la Protezione e la Ricerca Ambientale for the sea level data. We would like to thank the Progetto Mediterranea for their fruitful scientific and technological collaboration and support in the dissemination of SeaConditions to Mediterranean users. We would also like to thank Studio Valla for the fruitful collaboration in the collection of user feedback on SeaConditions.

Edited by: R. Archetti

Reviewed by: two anonymous referees

\section{References}

Almirall, E. and Wareham, J.: Living Labs: Arbiters of Mid- and Ground-Level Innovation, Technol. Anal. Strateg., 23, 87-102, doi:10.1080/09537325.2011.537110, 2011.

Bignami, F., Marullo, S., Santoleri, R., and Schiano, M. E.: Longwave radiation budget in the Mediterranean Sea, J. Geophys. Res.-Oceans, 100, 2501-2514, 1995.

Castellari, S., Pinardi, N., and Leaman, K.: A model study of air-sea interactions in the Mediterranean Sea, J. Mar. Syst., 18, 89-114, 1998.

Chesbrough, H. W.: Open Innovation: The new imperative for creating and profiting from technology, Boston, Harvard Business School Press, Boston, USA, 2003.

De Dominicis, M., Pinardi, N., Zodiatis, G., and Lardner, R.: MEDSLIK-II, a Lagrangian marine surface oil spill model for short-term forecasting - Part 1: Theory, Geosci. Model Dev., 6, 1851-1869, doi:10.5194/gmd-6-1851-2013, 2013.

Dobricic, S. and Pinardi, N.: An oceanographic three-dimensional variational data assimilation scheme, Ocean Model., 22, 89-105, 2008.

Dobricic, S., Pinardi, N., Adani, M., Tonani, M., Fratianni, C., Bonazzi, A., and Fernandez, V.: Daily oceanographic analyses by Mediterranean Forecasting System at the basin scale, Ocean Sci., 3, 149-157, doi:10.5194/os-3-149-2007, 2007.

Guarnieri, A., Oddo, P., Pastore, M., Pinardi, N., and Ravaioli, M.: The Adriatic Basin Forecasting System: new model and system development, in: Coastal to Global Operational Oceanography: Achievements and Challenges, edited by: Dahlin, H., Fleming, N. C., and Petersson, S. E., Proceeding of 5th EuroGOOS Conference, 20-22 May 2008, Exeter, 184-190, 2010.

Lecci, R., Coppini, G., Cretì, S., Turrisi, G., D’Anca, A., Palazzo, C., Aloisio, G., Fiore, S., Bonaduce, A., Mannarini, G., Kumkar, Y., Ciliberti, S. A., Federico, I., Agostini, P., Bonarelli, R., Martinelli, S., Marra, P., Scalas, M., Tedesco, L., Rollo, D., Cavallo, A., Tumolo, A., Monacizzo, T., Spagnulo, M., Pinardi, N., Fazioli, L., Olita, A., Cucco, A., Sorgente, R., Tonani, M., and Drudi, M.: SeaConditions: Present and future sea conditions for safer navigation, in: Proceedings of OCEANS'15 MTS/IEEE GENOVA, 18-21 May 2015, Genova, doi:10.1109/OCEANSGenova.2015.7271764, 2015.

Mannarini, G., Pinardi, N., Coppini, G., Oddo, P., and Iafrati, A.: VISIR-I: small vessels - least-time nautical routes using wave 
forecasts, Geosci. Model Dev., 9, 1597-1625, doi:10.5194/gmd9-1597-2016, 2016.

Oddo, P., Pinardi, N., and Zavatarelli, M.: A numerical study of the interannual variability of the Adriatic Sea (2000-2002), Sci. Total Environ., 353, 39-56, 2005.

Oddo, P., Pinardi, N., Zavatarelli, M., and Colucelli, A.: The Adriatic Basin forecasting system, Acta Adriat., 47, 169-184, 2006.

Oddo, P., Adani, M., Pinardi, N., Fratianni, C., Tonani, M., and Pettenuzzo, D.: A nested Atlantic-Mediterranean Sea general circulation model for operational forecasting, Ocean Sci., 5, 461-473, doi:10.5194/os-5-461-2009, 2009.

Olita, A., Sorgente, R., Natale, S., Gabersek, S., Ribotti, A., Bonanno, A., and Patti, B.: Effects of the 2003 European heatwave on the Central Mediterranean Sea: surface fluxes and the dynamical response, Ocean Sci., 3, 273-289, doi:10.5194/os-3273-2007, 2007.

Olita, A., Dobricic, S., Ribotti, A., Fazioli, L., Cucco, A., Dufau, C., and Sorgente, R.: Impact of SLA assimilation in the Sicily Channel Regional Model: model skills and mesoscale features, Ocean Sci., 8, 485-496, doi:10.5194/os-8-485-2012, 2012.

Pinardi, N., Allen, I., Demirov, E., De Mey, P., Korres, G., Lascaratos, A., Traon, P. Y., Maillard, C., Manzella, G., and Tziavos, C.: The Mediterranean ocean forecasting system: first phase of implementation (1998-2001), Ann. Geophys., 21, 3-20, 2003.
Raicich, F.: Note on flow rates of the Adriatic rivers, Tech. Rep., CNR Istituto Talassografico Sperimentale, Trieste, RF 02/94, 8 pp., 1994.

Sorgente, R., Drago, A. F., and Ribotti, A.: Seasonal variability in the Central Mediterranean Sea circulation, Ann. Geophys., 21, 299-322, 2003.

Sorgente, R., Olita, A., Oddo, P., Fazioli, L., and Ribotti, A.: Numerical simulation and decomposition of kinetic energy in the Central Mediterranean: insight on mesoscale circulation and energy conversion, Ocean Sci., 7, 503-519, doi:10.5194/os-7-5032011, 2011.

Tolman, H.: User Manual and system documentation of WAVEWATCH III version 3.14, NOAA/NWS/NCEP/MMAB Technical Note 276, 194 pp., 2009.

Tonani, M., Pinardi, N., Dobricic, S., Pujol, I., and Fratianni, C.: A high-resolution free-surface model of the Mediterranean Sea, Ocean Sci., 4, 1-14, doi:10.5194/os-4-1-2008, 2008.

Tonani, M., Pinardi, N., Fratianni, C., Pistoia, J., Dobricic, S., Pensieri, S., de Alfonso, M., and Nittis, K.: Mediterranean Forecasting System: forecast and analysis assessment through skill scores, Ocean Sci., 5, 649-660, doi:10.5194/os-5-649-2009, 2009.

Von Hippel, E.: Lead users: a source of novel product concepts, Manage. Sci., 32, 791-805, doi:10.1287/mnsc.32.7.791, 1986. 